Pacific Journal of Mathematics

AN INTERPOLATION THEOREM FOR ANALYTIC FAMILIES
OF OPERATORS ACTING ON CERTAIN $H^{p}$ SPACES 


\section{AN INTERPOLATION THEOREM FOR ANALYTIC FAMILIES OF OPERATORS ACTING ON CERTAIN $H^{p}$ SPACES}

\section{EUGENIO HERNÁNDEZ}

The main objective of this paper is to obtain an interpolation theorem for families of operators acting on atomic $H^{p}$ spaces, $0<p \leq 1$. We prove that if $0<p_{0}<p_{1} \leq 1$ and $\left\{T_{z}\right\}, z \in \bar{S}=\{z \in \mathbf{C} / 0 \leq \operatorname{Real} z$ $\leq 1\}$, is an analytic and admissible family of linear transformations such that $T_{j+l y}$ maps $H^{p_{j}}$ into $L^{p_{j}}$, where $-\infty<y<\infty$, with norm not exceeding $A_{j}, j=0,1$, then for all $\theta, 0 \leq \theta \leq 1, T_{\theta}$ maps $H^{r}$ into $L^{r}$ with norm not exceeding $c A_{0}^{1-\theta} A_{1}^{\theta}$, where $1 / r=(1-\theta) / p_{0}+\theta / p_{1}$.

1. Introduction. For $x \in \mathbf{R}^{n}$ and $\alpha=\left(\alpha_{1}, \ldots, \alpha_{n}\right)$ a multi-index, with $\alpha_{j}$ a natural number, $j=1, \ldots, n$, we write $x^{\alpha}=x_{1}^{\alpha_{1}} \cdots x_{n}^{\alpha_{n}}$ and $|\alpha|=\alpha_{1}+\cdots+\alpha_{n}$. For $0<p \leq 1$ and an integer $s \geq[n(1 / p-1)]^{1}$ we say that a function $a(x)$ is a $(p, s)$-atom if:

(i) the support of $a$ is contained in a ball $B \subset \mathbf{R}^{n}$.

(ii) $|a(x)| \leq|B|^{-1 / p}$ for all $x \in \mathbf{R}^{n}(|B|$ denotes the measure of $B)$.

(iii) $\int_{\mathbf{R}^{n}} a(x) x^{\alpha} d x=0$ for all multi-indexes $\alpha$ with $|\alpha| \leq s$.

The space $H^{p, s}\left(\mathbf{R}^{n}\right)$ consists of those linear functionals $f$ defined on an appropriate Lipschitz class of test functions (see [6] for details, where more general atoms are also considered) that can be expressed in the form $f=\sum_{i=1}^{\infty} \lambda_{i} a_{i}$, where the $a_{l}$ are $(p, s)$-atoms and $\sum_{i=1}^{\infty}\left|\lambda_{i}\right|^{p}<\infty$. The quasi-norm of $f$ is defined to be the infimum of the numbers $\left(\sum_{l=1}^{\infty}\left|\lambda_{i}\right|^{p}\right)^{1 / p}$ taken over all the above representations of $f$; it is denoted by $\|f\|_{H^{p, s .}}$. For fixed $p$, the spaces $H^{p, s}$ are independent of $s$ and their quasi-norms are equivalent (for this and the relation of these spaces to the classical $H^{p}$ spaces see [6]). For this reason we shall denote each of these equivalent spaces by $H^{p}$ and \|\|$_{H^{p}}$ will denote the quasi-norm.

Another aspect of this theorem that we must make precise is the type of transformations we are dealing with.

A function $\Phi$ defined on $\bar{S}$, the closure of $S$, is said to be of admissible growth if there exist positive constants $B$ and $b, b<\pi$, such that

$$
\Phi(z) \leq B e^{b|\operatorname{Im} z|}
$$

for all $z \in \bar{S}$.

\footnotetext{
${ }^{1}[t]$ denotes the integer part of the non-negative real number $t$.
} 
A family of linear transformations $\left\{T_{z}\right\}, z \in \bar{S}$, each mapping simple functions to measurable functions on $\mathbf{R}^{n}$, is called analytic if the function

$$
z \rightarrow \int_{\mathbf{R}^{n}}\left(T_{z} \varphi\right) \psi d x
$$

is analytic for each pair of simple functions $\varphi$ and $\psi$ on $\mathbf{R}^{n}$.

This family is said to be admissible if the function $\log \left\|T_{z} \varphi\right\|_{1}$ is of admissible growth for each simple $\varphi$ on $\mathbf{R}^{n}$.

We are now ready to state the main theorem.

THEOREM I. Let $0<p_{0}<p_{1} \leq 1$ and $\left\{T_{z}\right\}, z \in \bar{S}$, be an analytic and admissible family of linear transformations. We suppose

$$
\left\|T_{j+i, y} f\right\|_{L^{p_{j}}} \leq A_{j}\|f\|_{H^{p_{j}}}, \quad j=0,1,
$$

for all $f \in H^{p_{j}} \cap L^{p_{j}}$, where $-\infty<y<\infty$ and $A_{j}, j=0,1$, are positive constants.

Then, for each $\theta, 0 \leq \theta \leq 1$, we have

$$
\left\|T_{\theta} f\right\|_{L^{r}} \leq c A_{0}^{1-\theta} A_{1}^{\theta}\|f\|_{H^{r}}
$$

for all $f \in H^{r}$, where $1 / r=(1-\theta) / p_{0}+\theta / p_{1}$ and $c$ depends only on the dimension $n$ and the quasi-norm used in $H^{r}$.

A similar theorem for transformations acting on $H^{p}$ of the unit disk was proved by E. M. Stein and G. Weiss [5]. ${ }^{2}$

2. Proof of Theorem I. Due to the equivalence of the spaces $H^{p, s}$ for $p$ fixed, Theorem I follows from the theorem below.

THEOREM II. Let $0<p_{0}<p_{1} \leq 1$ and $s$ an integer satisfying $s \geq$ $\left[n\left(1 / p_{0}-1\right)\right]$. Let $\left\{T_{z}\right\}, z \in \bar{S}$, be an analytic and admissible family of linear transformations such that

$$
\left\|T_{j+i y} f\right\|_{L^{p_{j}}} \leq A_{j}\|f\|_{H^{p_{j}, s}}, \quad j=0,1,
$$

for all $f \in H^{p_{j}, s} \cap L^{p_{j}}$, where $-\infty<y<\infty$ and $A_{j}, j=0,1$, are positive constants.

\footnotetext{
${ }^{2}$ In [4] E. M. Stein proved a similar theorem for transformations acting on $L^{p}$ spaces.
} 
Then, for each $\theta, 0 \leq \theta \leq 1$, we have

$$
\left\|T_{\theta} f\right\|_{L^{r}} \leq A_{0}^{1-\theta} A_{1}^{\theta}\|f\|_{H^{r, s}}
$$

for all $f \in H^{r, s}$, where $1 / r=(1-\theta) / p_{0}+\theta / p_{1}$.

Three lemmas are used to prove this theorem.

LeMMA 1. Let $\Phi: S \rightarrow \mathbf{R}$ be an upper semi-continuous function of admissible growth and subharmonic in $S$. Then for $z_{0}=x_{0}+i y_{0} \in S$ we have

$$
\begin{aligned}
\Phi\left(z_{0}\right) \leq & \int_{-\infty}^{\infty} \Phi\left(i\left[y_{0}+y\right]\right) \omega\left(1-x_{0}, y\right) d y \\
& +\int_{-\infty}^{\infty} \Phi\left(1+i\left[y_{0}+y\right]\right) \omega\left(x_{0}, y\right) d y
\end{aligned}
$$

where

$$
\omega(x, y)=\frac{1}{2} \frac{\sin \pi x}{\cos \pi x+\cosh \pi y} .
$$

For a proof see [5].

LEMMA 2. If $V(z)$ is an analytic function mapping $S$ into $L^{1}$, then, for any positive $c \leq 1, \Phi(z)=\int_{\mathbf{R}^{n}}|V(z)|^{c} d x$ is continuous and $\log \Phi(z)$ is subharmonic.

The proof can be found in [5].

LEMMA 3. Let $0<p_{0}<p_{1} \leq 1$ and $s$ an integer satisfying $s \geq$ $\left[n\left(1 / p_{0}-1\right)\right]$. Let $a$ be an $(r, s)$-atom, where $1 / r=(1-\theta) / p_{0}+\theta / p_{1}$ and $0 \leq \theta \leq 1$.

Then there exists an analytic function $h(z)$ mapping $S$ into $L^{1}$ such that

$$
\|h(i y)\|_{H^{p_{0}, s}} \leq 1, \quad\|h(1+i y)\|_{H^{p_{1}, s}} \leq 1
$$

for any real $y$ and $h(\theta)=a$.

Proof. Let $B$ be the support of $a$ and define $h(z)=|B|^{\alpha(z)} a$ where $\alpha(z)=\left(1 / r-1 / p_{0}\right)(1-z)+\left(1 / r-1 / p_{1}\right) z$. Observe that

$$
\alpha(\theta)=0 .
$$

$\operatorname{Real}\{\alpha(i y)\}=1 / r-1 / p_{0}$ for all real $y$.

$$
\operatorname{Real}\{\alpha(1+i y)\}=1 / r-1 / p_{1} \text { for all real } y \text {. }
$$


Using the fact (easily verified) that $a$ belongs to $H^{p_{0}, s}$ with quasi-norm not exceeding $|B|^{1 / p_{0}-1 / r}$ and (2.3), we obtain

$$
\|h(i y)\|_{H^{p_{0 . s}}}=|B|^{1 / r-1 / p_{0}}\|a\|_{H^{p_{0}, s}} \leq 1 .
$$

Similarly, $\|h(1+i y)\|_{H^{p \mid . s}} \leq 1$. Finally, (2.2) implies $h(\theta)=a$.

Proof of Theorem II. Let $a$ be an $(r, s)$-atom and denote by $h(z)$ the analytic function associated with $a$ as in Lemma 3. Choose an integer $k$ such that $k p_{0}>r$ and $g$ a positive simple function with $\|g\|_{k^{\prime}} \leq 1$ where $1 / k+1 / k^{\prime}=1$. Consider

$$
\Phi(z)=\int_{\mathbf{R}^{n}}\left|[g(x)]^{\beta(z)}\right|\left|\left(T_{z} h(z)\right)(x)\right|^{r / k} d x
$$

where

$$
\beta(z)=k^{\prime}-\frac{k^{\prime} r}{k}\left(\frac{1-z}{p_{0}}+\frac{z}{p_{1}}\right)
$$

Note that

$$
\begin{gathered}
\beta(\theta)=1 . \\
\operatorname{Real} \beta(i y)=\frac{k^{\prime}\left(k p_{0}-r\right)}{k p_{0}} \quad \text { for all real } y . \\
\operatorname{Real} \beta(1+i y)=\frac{k^{\prime}\left(k p_{1}-r\right)}{k p_{1}} \quad \text { for all real } y .
\end{gathered}
$$

Using Hölder's inequality with index $k p_{0} / r>1$, (2.6) and the fact that the $L^{k^{\prime}}$ norm of $g$ is bounded by 1 , we obtain

$$
|\Phi(i y)| \leq\left\{\int_{\mathbf{R}^{n}}\left|\left(T_{l y} h(i y)\right)(x)\right|^{p_{0}} d x\right\}^{r / k p_{0}} .
$$

Using this inequality, hypothesis (2.1) and Lemma 3, we obtain

$$
|\Phi(i y)| \leq A_{0}^{r / k}\|h(i y)\|_{H^{p_{0}, s}} \leq A_{0}^{r / k} .
$$

Similarly, using Hölder's inequality with index $k p_{1} / r>1$, we obtain

$$
|\Phi(1+i y)| \leq A_{1}^{r / k} \text {. }
$$

Lemma 2 implies that $\log \Phi(z)$ is subharmonic. Consequently, Lemma 1 and inequalities (2.8) and (2.9) imply ${ }^{3}$

$$
\log \Phi(\theta) \leq \log A_{0}^{r(1-\theta) / k} A_{1}^{r \theta / k} .
$$

${ }^{3}$ Observe that $\int_{-\infty}^{\infty} \omega(1-\theta, y) d y=1-\theta$ and $\int_{-\infty}^{\infty} \omega(\theta, y) d y=\theta$. 
Noticing that $\Phi(\theta)=\int_{\mathbf{R}^{n}} g(x)\left|\left(T_{\theta} a\right)(x)\right|^{r / k} d x$, the above inequality, together with

$$
\begin{aligned}
& \left\{\int_{\mathbf{R}^{n}}\left|\left(T_{\theta} a\right)(x)\right|^{r} d x\right\}^{1 / k} \\
& \quad=\sup \left\{\int_{\mathbf{R}^{n}}\left|\left(T_{\theta} a\right)(x)\right|^{r / k} g(x) d x \mid, g \geq 0, g \text { simple, }\|g\|_{k^{\prime}} \leq 1\right\},
\end{aligned}
$$

implies

$$
\left\{\int_{\mathbf{R}^{n}}\left|\left(T_{\theta} a\right)(x)\right|^{r} d x\right\}^{1 / k} \leq A_{0}^{r(1-\theta) / k} A_{1}^{r_{\theta} / k},
$$

which proves Theorem II for atoms. From here the theorem follows from the definition of $H^{r, s}$ in terms of atoms.

3. Remarks. We start with some historical remarks. $H^{p}$ spaces can be defined on spaces of homogeneous type (see [1]). The main difference with the $H^{p}$ spaces that we consider here is that (iii) in the definition of an atom is replaced by $\int a(x) d x=0$. The reader will have no trouble verifying that Theorem I can be extended to include the homogeneous type case. In this context R. Macías proved in his dissertation (1973-1974) a particular case of Theorem I (see [3]). An interpolation theorem for linear operators acting on $H^{p}\left(\mathbf{R}^{n}\right)$ was published in 1974 by C. Fefferman, N. Rivière and Y. Sagher ([2]). Their proof uses the real method of interpolation and is quite different from Macías' proof. For this reason, it is unfortunate that Macias' dissertation was never published. Our work fills the gap left by the unpublished work of R. Macías.

It is worthwhile to notice that the fact proved by $M$. Taibleson and $\mathrm{G}$. Weiss ([6]) concerning the equivalence of $H^{p, s}$ spaces for fixed $p$ plays an important role in the proof of Theorem I.

Theorem I has several extensions. First, $\mathbf{R}^{n}$ can be replaced by any other space for which an atomic theory similar to the one described above can be defined. Second, condition (1.1) can be replaced by

$$
\left\|T_{j+i y} f\right\|_{L^{q_{j}}} \leq A_{j}(y)\|f\|_{H^{p_{j}}}, \quad j=0,1,
$$

where $0<q_{j}<\infty, \log A_{j}(y) \leq C_{j} e^{d_{j}|\nu|}, 0<d_{j}<\pi$ and $C_{j}>0, j=0,1$. Then, it is easy to check that the conclusion of the theorem becomes

$$
\left\|T_{\theta} f\right\|_{L^{q}} \leq C\|f\|_{H^{r}}
$$

where $C$ depends on $\theta, p_{J}, q_{j}, C_{j}, d_{j}(j=0,1)$ and the dimension $n$ and $1 / q=(1-\theta) / q_{0}+\theta / q_{1}$. 


\section{REFERENCES}

[1] R. Coifman and G. Weiss, Extensions of Hardy spaces and their use in analysis, Bull. Amer. Math. Soc., 83, No. 4 (1977), 569-645.

[2] C. Fefferman, N. Rivière and Y. Sagher, Interpolation between $H^{p}$ spaces, the real method, Trans. Amer. Math. Soc., 19 (1974), 75-82.

[3] R. A. Macias, Interpolation theorems on generalized Hardy spaces, Dissertation, Washington Univ., St. Louis, Missouri (1974).

[4] E. M. Stein, Interpolation of linear operators, Trans. Amer. Math. Soc., 83 (1956), 482-492.

[5] E. M. Stein and G. Weiss, On the interpolation of analytic families of operators acting on $H^{p}$ spaces, Tôhoku Math. J., 8 (1957), 318-339.

[6] M. H. Taibleson and G. Weiss, The molecular characterization of certain Hardy spaces, Astérisque, 77 (1980), 67-149.

Received March 8, 1982 and in revised form December 16, 1982. This work is part of the author's dissertation which was completed at Washington University in St. Louis. The author expresses deep appreciation to his advisors, Richard Rochberg and Guido Weiss, for their support.

WASHINGTON UNIVERSITY

ST. LouIs, MO 63130 


\section{PACIFIC JOURNAL OF MATHEMATICS \\ EDITORS}

DONALD BABBITT (Managing Editor)

University of California

Los Angeles, CA 90024

Hugo RossI

University of Utah

Salt Lake City, UT 84112

C. C. MOOre and ARThur OGus

University of California

Berkeley, CA 94720
J. Dugundi

Department of Mathematics

University of Southern California

Los Angeles, CA 90089-1113

R. FINN and H. SAMELSON

Stanford University

Stanford, CA 94305

ASSOCIATE EDITORS

$\begin{array}{lllll}\text { R. Arens } & \text { E. F. Beckenbach } & \text { B. H. Neumann } & \text { F. Wolf } & \text { K. Yoshida }\end{array}$ $(1906-1982)$

\section{SUPPORTING INSTITUTIONS}

UNIVERSITY OF ARIZONA

UNIVERSITY OF BRITISH COLUMBIA

CALIFORNIA INSTITUTE OF TECHNOLOGY

UNIVERSITY OF CALIFORNIA

MONTANA STATE UNIVERSITY

UNIVERSITY OF NEVADA, RENO

NEW MEXICO STATE UNIVERSITY

OREGON STATE UNIVERSITY
UNIVERSITY OF OREGON

UNIVERSITY OF SOUTHERN CALIFORNIA

STANFORD UNIVERSITY

UNIVERSITY OF HAWAII

UNIVERSITY OF TOKYO

UNIVERSITY OF UTAH

WASHINGTON STATE UNIVERSITY

UNIVERSITY OF WASHINGTON 


\section{Pacific Journal of Mathematics}

\section{Vol. 110, No. $1 \quad$ September, 1984}

Wojciech Abramczuk, A class of surjective convolution operators $\ldots \ldots \ldots \ldots 1$

K. Adachi, Extending bounded holomorphic functions from certain

subvarieties of a weakly pseudoconvex domain $\ldots \ldots \ldots \ldots \ldots \ldots$

Malvina Florica Baica, An algorithm in a complex field and its application

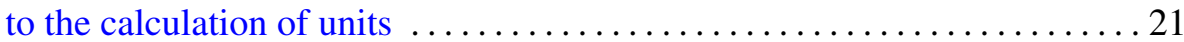

Giuliana Bianchi and Robert Cori, Colorings of hypermaps and a

conjecture of Brenner and Lyndon $\ldots \ldots \ldots \ldots \ldots \ldots \ldots \ldots \ldots \ldots \ldots \ldots$

Ronald James Evans, Determinations of Jacobsthal sums . . .......... 49

Leslie Foged, Characterizations of $\aleph$-spaces .................... 59

Nassif A. Ghoussoub and Paulette Saab, Weak compactness in spaces of

Bochner integrable functions and the Radon-Nikodým property . . . . . . 65

J. Gómez Gil, On local convexity of bounded weak topologies on Banach

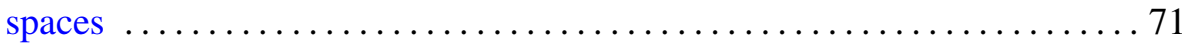

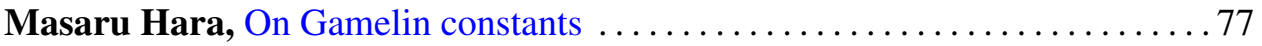

Wilfried Hauenschild, Eberhard Kaniuth and Ajay Kumar, Harmonic

analysis on central hypergroups and induced representations $\ldots \ldots \ldots 83$

Eugenio Hernandez, An interpolation theorem for analytic families of

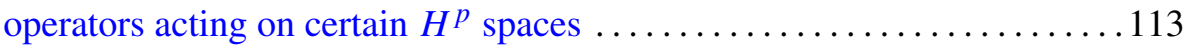

Thomas Alan Keagy, On "Tauberian theorems via block-dominated

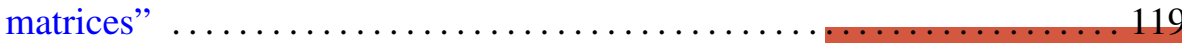

Thomas Landes, Permanence properties of normal structure $\ldots \ldots \ldots \ldots \ldots 125$

Daniel Henry Luecking, Closed ranged restriction operators on weighted

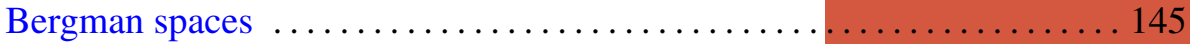

Albert Thomas Lundell, The $p$-equivalence of $\mathrm{SO}(2 n+1)$ and $\mathrm{Sp}(n) \ldots \ldots 161$

Mark D. Meyerson, Remarks on Fenn's "the table theorem" and Zaks' "the chair theorem" ..................................... 167

Marvin Victor Mielke, Homotopically trivial toposes . . . . . . . . . . 171

Gerard J. Murphy, Hyperinvariant subspaces and the topology on Lat A . . 183

Subhashis Nag, On the holomorphy of maps from a complex to a real manifold

Edgar Milan Palmer and Robert William Robinson, Enumeration of self-dual configurations ................................. 203

John J. Walsh and David Clifford Wilson, Continuous decompositions

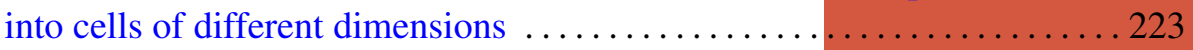

Walter John Whiteley, Infinitesimal motions of a bipartite framework .....233 